University of Wollongong

Research Online

Faculty of Engineering - Papers (Archive)

Faculty of Engineering and Information

Sciences

2004

\title{
Verification of Monte Carlo calculations in fast neutron therapy using silicon microdosimetry
}

I. Cornelius

University of Wollongong, iwan@uow.edu.au

Anatoly B. Rosenfeld

University of Wollongong, anatoly@uow.edu.au

Follow this and additional works at: https://ro.uow.edu.au/engpapers

Part of the Engineering Commons

https://ro.uow.edu.au/engpapers/27

\section{Recommended Citation}

Cornelius, I. and Rosenfeld, Anatoly B.: Verification of Monte Carlo calculations in fast neutron therapy using silicon microdosimetry 2004.

https://ro.uow.edu.au/engpapers/27

Research Online is the open access institutional repository for the University of Wollongong. For further information contact the UOW Library: research-pubs@uow.edu.au 


\title{
Verification of Monte Carlo Calculations in Fast Neutron Therapy Using Silicon Microdosimetry
}

\author{
Iwan M. Cornelius, Student Member, IEEE, and Anatoly B. Rosenfeld, Senior Member, IEEE
}

\begin{abstract}
Silicon microdosimetry measurements in Fast Neutron Therapy were simulated using the GEANT4 Monte Carlo toolkit. The possibility of using silicon microdosimeters for verification of Monte Carlo based treatment planning systems in hadron therapy is suggested.
\end{abstract}

Index Terms-Fast neutron therapy (FNT), microdosimetry, Monte Carlo.

\section{INTRODUCTION}

$\mathbf{T}$ HE biological effects of densely ionising radiation, as encountered in hadron therapy, cannot be predicted from the absorbed dose and several radiobiological models have been developed to address this issue [1]-[5]. These models require detailed information on the nature of the mixed radiation field in order to calculate biological effect. This information is readily available from Monte Carlo calculations which may accurately model the mixed radiation field present with a patient during hadron therapy. Monte Carlo calculations for treatment planning purposes may be performed in a reasonable time by exploiting parallel computing techniques [6].

The ideal method for treatment plan verification is to measure the three-dimensional (3-D) distribution of a biological end point, such as cell survival, obtained in a heterogeneous phantom. A preliminary study of the verification of carbon ion therapy treatment planning with two-dimensional (2-D) cell survival distributions is given in [7]. These studies are time consuming and dosimetry measurements are necessary to routinely verify the model of the mixed radiation field in the patient. The measurement of absorbed dose alone may not be suitable if dose is not indicative of biological effect. Ideally, one would like a spectroscopic technique to measure the energy spectrum of all charged particles at the point of interest. This paper proposes the use of silicon microdosimeters which are sensitive to the LET spectrum of the charged particles.

Silicon microdosimetry was initially developed in the space radiation effects community [8] and was subsequently applied to conventional microdosimetry studies in hadron therapy applications [9]. A prototype silicon microdosimeter was developed which consists of an array of microscopic pn junctions fabricated on varying thicknesses of silicon-on-insulator (SOI) wafers. This array is reverse biased (at $10 \mathrm{~V}$ ) and coupled to a charge sensitive preamplifier which produces a voltage pulse

Manuscript received November 5, 2003; revised February 13, 2004. This work was supported by the Australian Institute of Nuclear Science and Engineering.

The authors are with the University of Wollongong, Wollongong, NSW 2522, Australia (e-mail: ic02@uow.edu.au).

Digital Object Identifier 10.1109/TNS.2004.829398 with amplitude proportional to the LET of each particle crossing the microdosimeter. Measurements were performed in Proton Therapy (PT) [10], Boron Neutron Capture Therapy (BNCT) [11], and Fast Neutron Therapy (FNT) [12]. In these studies the aim was to develop a silicon detector to replace the Tissue Equivalent Proportional Counter (TEPC). The aim of this paper is to simulate the silicon microdosimetry measurements performed by Bradley et al. [12], [13] in FNT to demonstrate the possibility of using such measurements to verify Monte Carlo calculations.

\section{METHODS}

\section{A. Experiment}

Measurements were conducted at the Gershenson Radiation Oncology Center (described in [14]). A probe containing the microdosimeter was placed at depth in a water phantom which was irradiated by the neutron beam. Ionization energy deposition events resulting from subsequent secondary charged particles were stored using a multichannel analyzer (MCA) spectrum. Energy calibration of the system is given in [10]. In these experiments a $2-\mu \mathrm{m}$-thick SOI device was used at $10 \mathrm{~cm}$ depth on the central axis of the beam, and a $10-\mu \mathrm{m}$ device was used at depths of $10 \mathrm{~cm}$ and $2.5 \mathrm{~cm}$.

These results were compared to microdosimetry spectra measured with a $2-\mu \mathrm{m}$ simulated diameter TEPC. The energy deposition event spectra recorded by the silicon microdosimeter were converted to dose weighted lineal energy spectra with the method described in [12]. After scaling of the mean chord length the microdosimetric spectra were seen to agree with that of the TEPC. This method of tissue equivalence correction is valid only if the secondary particle field is dominated by one species and only if "starting" particles, those resulting from neutron interactions in the silicon volume, can be ignored. The work suggested further investigation into the importance of neutron interactions in the silicon volume.

\section{B. Simulation}

The simulation was written in $\mathrm{C}++$ using classes which inherit behavior from base classes of the GEANT4 Monte Carlo toolkit [15]. These classes were used to model different aspects of the simulation such as; geometry, primary beam, physics of interaction, and actions carried out at the end of each particle history for analysing events. The geometry used in the simulation is given in Fig. 1 and follows closely the geometry of the experiment. Ion beam induced charge collection experiments with ion microbeams showed the charge collection efficiency of the microdosimeter varies with the location of the ion strike 


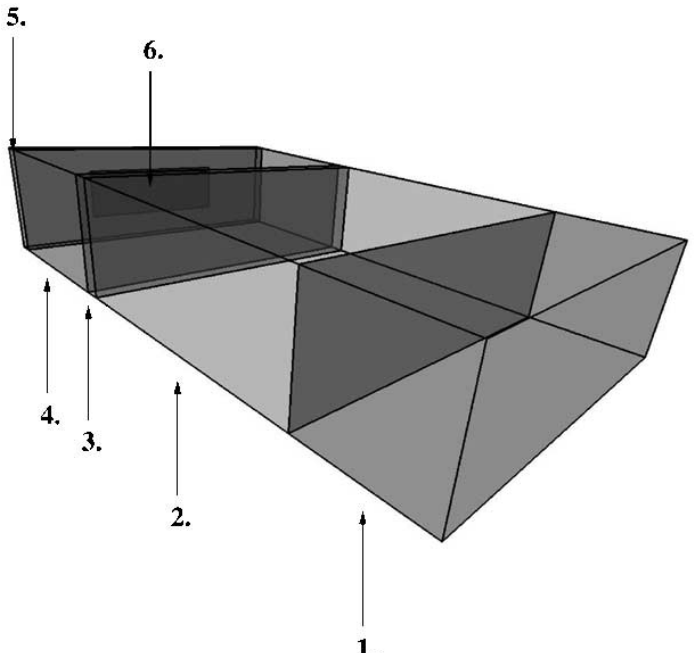

Fig. 1. Geometry used in the FNT simulation; 1. water phantom, 2. perspex envelope, 3. aluminum shielding, 4. perspex converter, 5. air gap and 6. silicon sensitive volume with 1 micron silicon oxide over-layer.

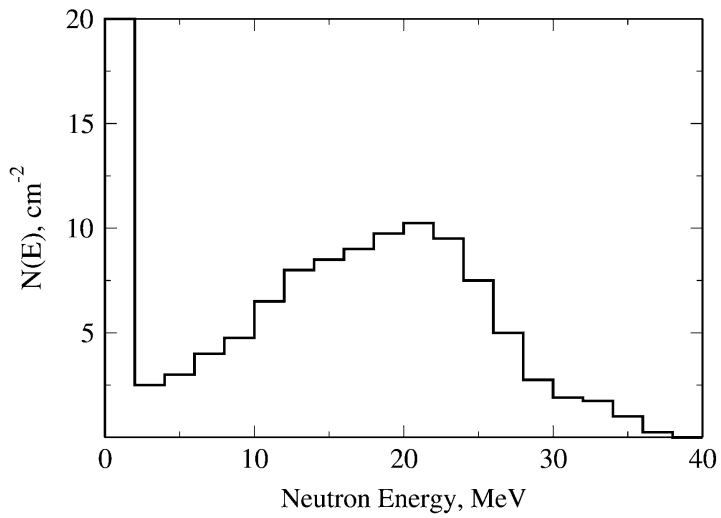

Fig. 2. Neutron energy distribution at the surface of the phantom at the Detroit FNT facility (taken from [14]).

on the device [16], [17]. At an operating bias of $10 \mathrm{~V}$ the average Charge Collection Efficiency (CCE) was found to be 0.8 for ions with LET in the range $20-820 \mathrm{keV} / \mu \mathrm{m}$. The silicon sensitive volume is consequently modeled as a single Right Angled Parallelapiped (RPP) of dimensions $4800 \times 1600 \times 10 \mu \mathrm{m}^{3}$ with a charge collection efficiency of 0.8 . The geometry of the device overlayer is simplified to a $\mathrm{SiO}_{2} \mathrm{RPP}$ of lateral dimensions identical to the sensitive volume and with a thickness of $1 \mu \mathrm{m}$. The $300 \mu \mathrm{m}$ air gap was modeled, as was the $3.5 \mathrm{~mm}$ perspex converter, $0.4 \mathrm{~mm}$ thick aluminum shield, $6 \mathrm{~mm}$ probe holder, and a $25 \mathrm{~mm}$ thickness of water corresponding to the depth of measurement. The lateral dimensions of these volumes were twice the width of the silicon sensitive volume. All materials are defined in terms of elemental compositions and in turn all elements are defined in terms of their isotopic composition.

The field size of the neutron beam used in the experiment was $10 \times 10 \mathrm{~cm}^{2}$, however for the simulation this was not feasible owing to a restriction on computation times. The neutron beam was modeled to be normally incident on the surface of the water phantom with lateral dimensions twice that of the microdosimeter array. The neutron energy distribution for the Detroit facility was obtained from a study by Kota et al. [14] and is shown in Fig. 2. For each neutron history of the simulation the initial energy was randomly sampled from this distribution. The

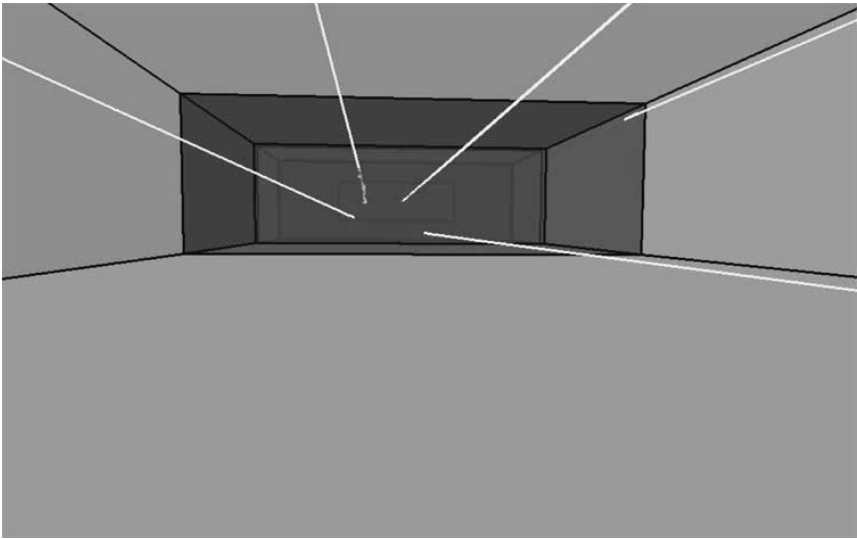

Fig. 3. View from within water phantom showing several neutron histories (green) and recoil protons (blue) generated in water phantom.

initial neutron position is randomly sampled on the surface of the phantom. This model of the primary beam ignores the production of the neutron beam and the interaction of the beam with modifying components. A total of $10^{8}$ neutrons were used for each simulation.

Each neutron is transported (see Fig. 3) taking into account elastic scattering on nuclei. In the neutron energy range $E>19.9 \mathrm{MeV}$ cross sections and end states are calculated using the G4LElastic model. For energies in the range $E<19.9 \mathrm{MeV}$ point wise evaluated nuclear cross section data is used and end states are calculated with the G4NeutronHPElastic model. A pre-equilibrium decay model, the G4PreCompoundModel, is used to model the inelastic scattering of neutrons with target nuclei in the energy range $E>19.9 \mathrm{MeV}$. For $E<19.9$ $\mathrm{MeV}$ the pointwise evaluated cross section data is used with the G4NeutronHPInelastic model. The final states considered for inelastic reactions are $(n A \rightarrow) n \gamma s$ (discrete continuum), $\quad n p, n d, n t, n^{3} \mathrm{He}, n \alpha, n d 2 \alpha, n t 2 \alpha, n 2 p, n 2 \alpha$, $n p \alpha, n 3 \alpha, 2 n, 2 n p, 2 n d, 2 n \alpha, 2 n 2 \alpha, n X, 3 n, 3 n p$,

$3 n \alpha, 4 n, p, p d, p \alpha, 2 p d, d \alpha, d 2 \alpha, d t, t, t 2 \alpha,{ }^{3} \mathrm{He}, \alpha, 2 \alpha, \quad$ and $3 \alpha$. Capture reactions are treated similarly, for $E>19.9$ $\mathrm{MeV}$ the G4LCapture is used and for $E<19.9 \mathrm{MeV}$ the G4NeutronHPCapture model is used.

Gamma photons are also transported through the simulation geometry. Interactions of gammas with atomic electrons are modeled with the G4ComptonScattering model for compton scattering and the photoelectric effect with the G4PhotoElectricEffect model.

Electrons are transported through the geometry taking into account scattering on atomic electrons (G4MultipleScattering model), ionization of atomic electrons (G4eIonization model), and Bremsstrahlung losses (G4eBremsstrahlung model).

Protons are transported taking into account elastic scatter interactions with the G4MultipleScattering model. Ionization of atomic electrons is also modeled (G4hLowEnergyIonization). Recoiling nuclei produced in elastic scatter interactions and reaction products from inelastics reactions also use these models. A pre-equilibrium decay model, the G4PreCompoundModel, is used to model the inelastic scattering of protons. A complete description of the physics models used in the simulations may be found in [15]. 


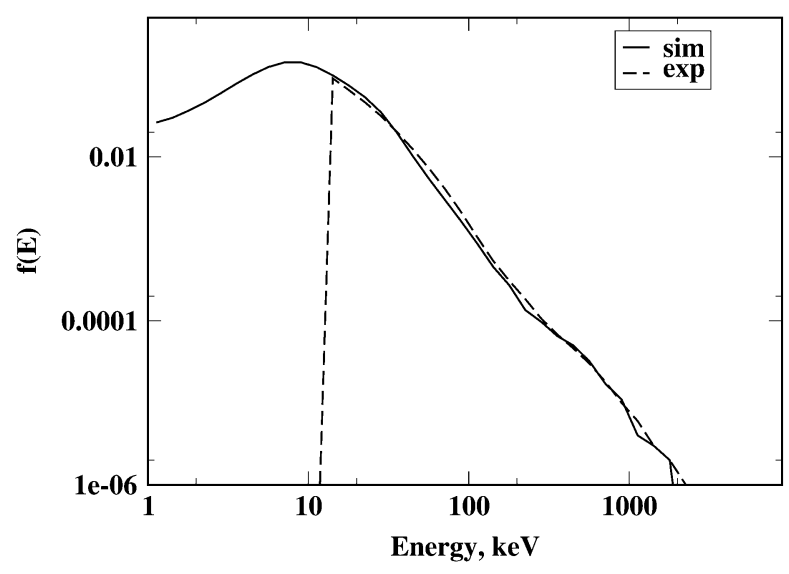

Fig. 4. Energy deposition event spectrum for $2 \mu \mathrm{m}$ SOI device.

If a charged particle is produced with a residual range in the material less than a default cut value of $10 \mu \mathrm{m}$ then the particle is not tracked. In this case the particle is assumed to deposit it's entire energy at the point of generation.

If a charged particle traverses the sensitive volume of the detector, then the ionization energy lost in the sensitive volume is calculated. Energy lost in the sensitive volume is assumed to be equal to energy deposited and the energy deposition event is tallied. In this situation the statistics of electron hole pair generation are ignored. For each particle crossing the sensitive volume the atomic number and mass of the particle is tallied. Whether the particle stopped in, crossed, or started in the sensitive volume is also determined.

An application was written in $\mathrm{C}++$ to analyze the output of these simulations. A frequency distribution of all energy deposition events, $f_{j}$, was formed. This distribution was convolved with a gaussian distribution of variance $\sigma=5 \mathrm{keV}$ to allow for the electronic noise of the detector electronics;

$$
f_{j}=\sum_{i=j-3 \sigma}^{j+3 \sigma} \frac{1}{\sqrt{2 \sigma^{2} \pi}} f_{i} \exp -\frac{\left(\epsilon_{j}-\epsilon_{i}\right)^{2}}{2 \sigma^{2}}
$$

Where $\epsilon_{i}$ is the energy value corresponding to bin $i$ of the frequency distribution.

As the magnitude of energy deposition can span several orders of magnitude the linear binned spectrum was logarithmically binned with 10 bins per decade, covering energy deposition from $1 \mathrm{keV}-5 \mathrm{MeV}$.

\section{RESUlTS AND DISCUSSION}

Experimental results are only observed above the low-noise threshold of $10 \mathrm{keV}$ of the microdosimeter probe. The MCA used in these experiments is known to exhibit anomolous behavior at high count rates of pulses with amplitude below the threshold level. This results in pileup of these pulses which register as counts above the threshold. Experiment and simulation results are normalized accordingly to events greater than $20 \mathrm{keV}$, twice the threshold level.

\section{A. 2 Micron Device}

Fig. 4 shows the experimental and simulated spectra of energy deposition events for the $2 \mu \mathrm{m}$ device. Fig. 5 shows spectra

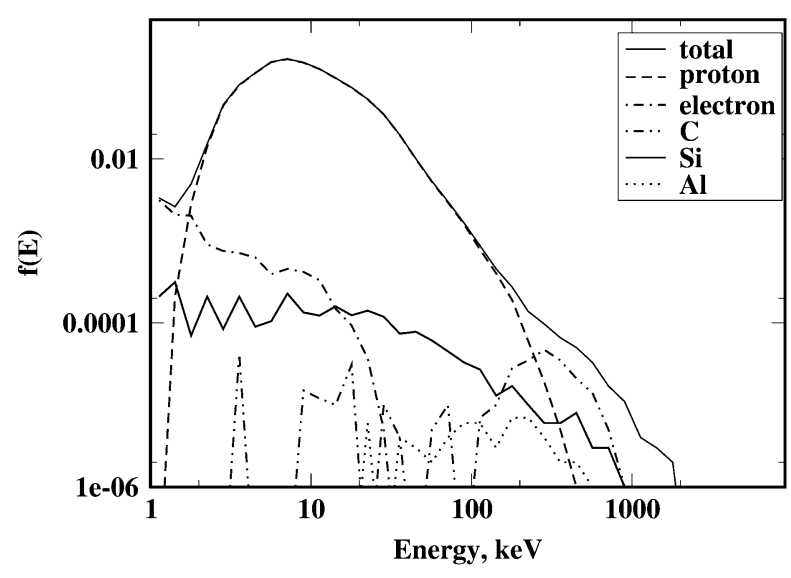

Fig. 5. Energy deposition event spectrum for $2 \mu \mathrm{m}$ SOI decomposed into spectra for each atomic number.

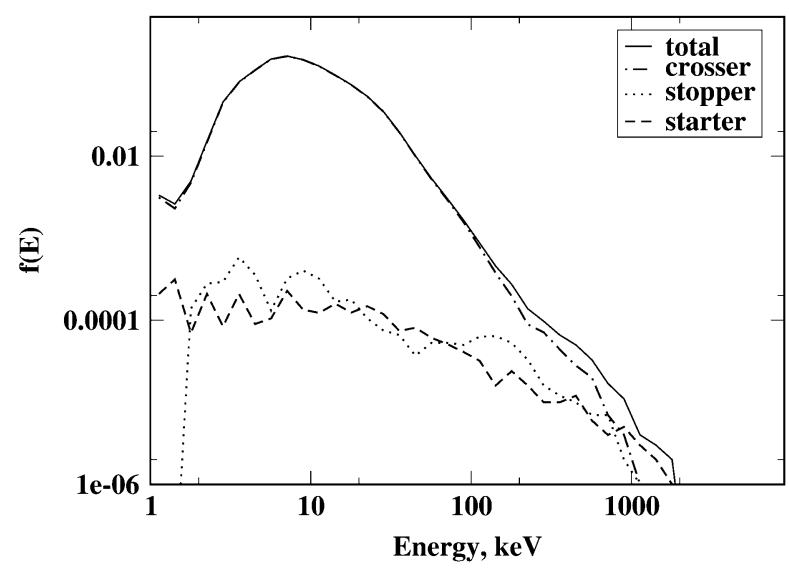

Fig. 6. Energy deposition event spectrum for $2 \mu \mathrm{m}$ SOI device decomposed into spectra for crossing, starting, and stopping particles.

formed for several components of the secondary charged particle field to understand the relative contribution to the total spectrum. The spectrum is dominated by recoil protons with a smaller contribution from carbon and other recoiling nuclei (some species omitted for clarity). A continuum corresponding to recoil silicon nuclei is clearly evident. At the peak of the distribution, the ratio of silicon recoil events to recoil proton events is approximately $1 \times 10^{-3}$.

To study the importance of neutron inelastic reactions in the silicon volume a spectrum is formed for particles which started in, stopped in, or crossed the microdosimeter. Results are shown in Fig. 6. These results show a significant contribution from starting and stopping particles, the significance of which is greater for higher energy deposition events. At the bin corresponding to the peak position, the ratio of stopping and starting events to crossing events is approximately $1 \times 10^{-3}$.

\section{B. 10 Micron Device}

Fig. 7 shows the experimental and theoretical spectrum of energy deposition events for the $10 \mu \mathrm{m}$ device. Again the results are normalized to the total number of events above $20 \mathrm{keV}$. In this situation there is significant discrepancy between experimental and theoretical curves in the interval $10-50 \mathrm{keV}$. Again, this spectrum is decomposed into relative spectra for each component of the charged particle field (see Fig. 8). This spectrum 


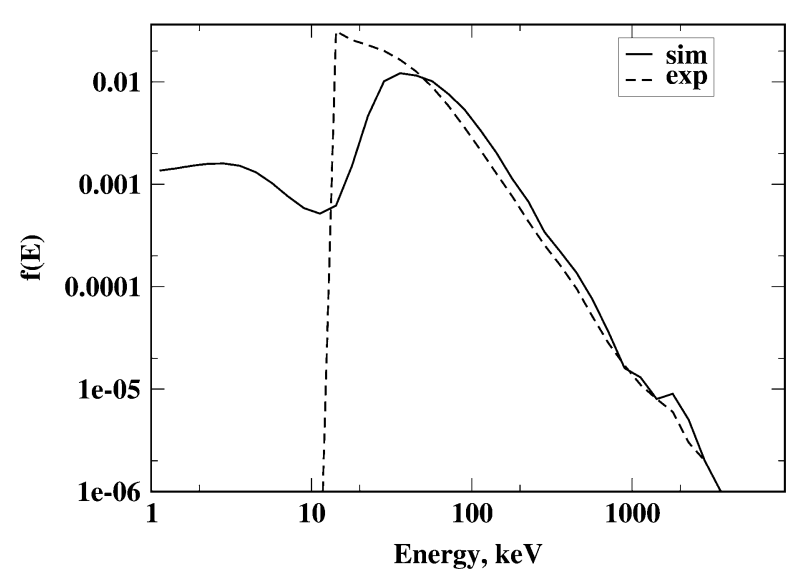

Fig. 7. Energy deposition event spectrum for $10 \mu \mathrm{m}$ SOI device.

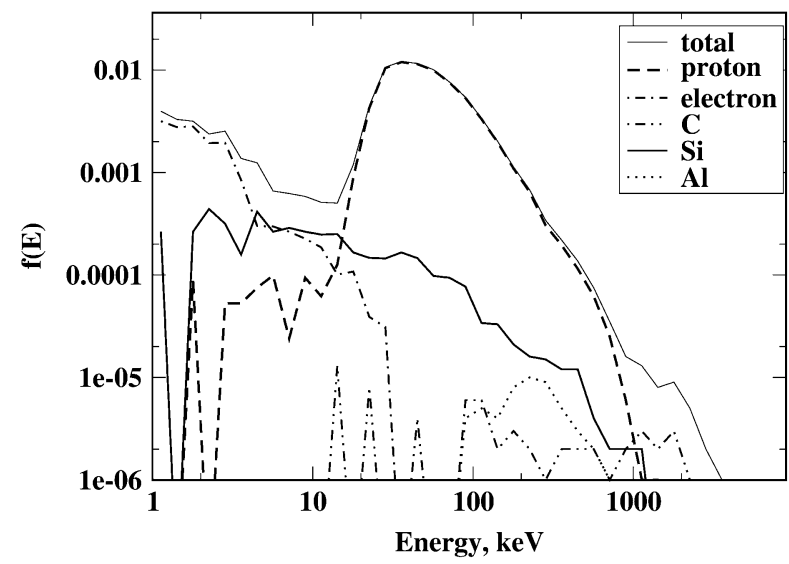

Fig. 8. Energy deposition event spectrum for $10 \mu \mathrm{m}$ SOI device decomposed into spectra for each atomic number.

is dominated by recoil protons with a smaller contribution from carbon ions and other recoils. The recoil silicon continuum is greater than the $2 \mu \mathrm{m}$ device. At the bin corresponding to the peak position, the ratio of silicon recoil events to recoil proton events is approximately $1 \times 10^{-2}$. This effect is a result of the increased device thickness and hence higher probability of neutron interaction in the sensitive volume. Additionally, the relative contribution of carbon recoils is less than that for the $2 \mu \mathrm{m}$ device. The discrepancy observed at lower energy deposition events may be attributed to the pileup of subthreshold events.

Again spectra were formed for particles which started in, stopped in, or crossed the microdosimeter. Results are shown in Fig. 9. At the bin corresponding to the peak position, the ratio of stopping and starting events to crossing events is approximately $1 \times 10^{-2}$. These results show an increase in the significance of starting and stopping particles compared to the $2 \mu \mathrm{m}$ device.

\section{CONCLUSION}

Simulations of silicon microdosimetry masurements in FNT were performed using the GEANT4 Monte Carlo toolkit. Simulation results were seen to compare favorably with experimental results. A discrepancy was observed for the $10 \mu \mathrm{m}$ device which is most likely due to the pileup of subthreshold events. Future simulations should be performed to model the beam production

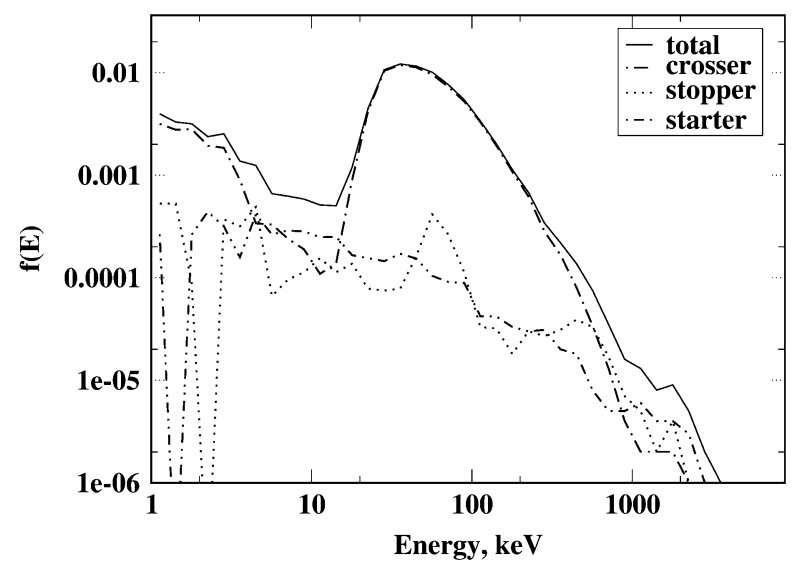

Fig. 9. Energy deposition event spectrum for $10 \mu \mathrm{m}$ SOI device decomposed into spectra for crossing, starting, and stopping particles.

and modification in detail and to investigate the anomolous behavior of the MCA near the threshold. To confirm the notion of pile up of subthreshold events, a pileup model should be incorporated into the simulation.

Simulation results were analyzed to study the importance of particles originating from neutron interactions in the silicon volume. This was seen to be significant for both 2 and $10 \mu \mathrm{m}$ results. If the silicon microdosimeters are being applied in the conventional sense of microdosimetry, these results show this effect may inhibit it's ability to provide tissue equivalent measurements. However, if the silicon microdosimeters are being applied in the sense of verifying the Monte Carlo calculation then neutron interactions in the silicon volume are not important.

This paper suggests how a nontissue equivalent detector may be used for the verification of Monte Carlo calculations in hadron therapy. Further development of silicon microdosimeters is in progress [12] as is the investigation of silicon detectors which are sensitive to ion track structure. Future experiments should be performed in heterogenous phantoms with materials of composition similar to tissues.

\section{ACKNOWLEDGMENT}

The authors would like to thank P. Bradley, previously of the Centre for Medical Radiation Physics, for the experimental data. The authors would also like to thank members of the GEANT4 online user's forum for their assistance.

\section{REFERENCES}

[1] R. Katz, R. Zachariah, F. A. Cucinotta, and C. Zhang, "Survey of cellular radiosensitivity parameters," Radiat. Res., vol. 140, pp. 356-365, 1994.

[2] H. Rossi and M. Zaider, Microdosimetry and Its Applications. Berlin, Germany: Springer-Verlag, 1996.

[3] M. Scholz, A. Kellerer, W. Kraft-Weyrather, and G. Kraft, "Computation of cell survival in heavy ion beams for therapy," Radiat. Environ. Biophys., vol. 36, pp. 59-66, 1997.

[4] R. Schulte, V. Bashkirov, S. Shchemelinin, G. Garty, R. Chechik, and A. Breskin, "Modeling of radiation action based on nanodosimetric event spectra," Phys. Med., vol. 17, no. Suppl: 1, pp. 177-180, 2001.

[5] M. Biaggi, F. Ballarini, W. Burkard, E. Egger, A. Ferrari, and A. Ottolenghi, "Physical and biophysical characteristics of a fully modulated $72 \mathrm{MeV}$ therapeutic proton beam: Model predictions and experimental data," Nucl. Instrum. Meth. B, vol. 159, pp. 89-100, 1999. 
[6] P. Bergstrom, A. Bielajew, W. Chandler, L. Cox, T. Daly, D. Garrett, B Guidry, C. H. Siantar, S. Hornstein, R. House, D. Jong, E. Moses, R. Patterson, J. Rathkopf, and A. C. von Wittenau, "Monte Carlo transport physics algorithms and variance reduction methods in the PEREGRINE dose calculation system," Med. Phys., pt. 1, vol. 25, no. 7, p. A186, 1998.

[7] M. Kramer, J. F. Wang, and W. Weyrather, "Biological dosimetry of complex ion radiation fields," Phys. Med. Biol., vol. 48, pp. 2063-2070, 2003.

[8] D. R. Roth, P. J. McNulty, W. J. Beauvais, R. A. Read, and E. G. Stassinopoulos, "Solid-state microdosimeter for radiation monitoring in spacecraft and avionics," IEEE Trans. Nucl. Sci., vol. 41, pp. 2118-2124, 1994.

[9] A. B. Rosenfeld, G. I. Kaplan, M. G. Carolan, B. J. Allen, R. Maughan, M. Yudelev, C. Kota, and J. Coderre, "Simultaneous macro-micro dosimetry with MOSFETs," IEEE Trans. Nucl. Sci., vol. 43, pp. 2693-2700, Dec. 1996

[10] A. Rosenfeld, P. Bradley, I. Cornelius, G. Kaplan, B. Allen, J. Flanz, M. Goitein, A. V. Meerbeeck, J. Schubert, J. Bailey, Y. Tabkada, A. Maruhashi, and Y. Hayakawa, "New silicon detector for microdosimetry applications in proton therapy," IEEE Trans. Nucl. Sci., vol. 47, pp. 1386-1394, Aug. 2000.
[11] P. Bradley, A. B. Rosenfeld, B. J. Allen, J. Corderre, and J. Capela, "Performance of silicon microdosimetry detectors in boron neutron capture therapy," Radiat. Res., vol. 151, pp. 235-243, 1999.

[12] P. Bradley, A. Rosenfeld, and M. Zaider, "Solid state microdosimetry," Nucl. Instrum. Meth. B, vol. 184, pp. 135-157, 2001.

[13] P. Bradley, "The Development of a Novel Silicon Microdosimeter for High LET Radiation Therapy," Ph.D. dissertation, University of Wollongong, Wollongong, Australia, 2000.

[14] T. D. Bohm, P. M. DeLuca, Jr., R. L. Maughan, D. T. L. Jones, and A. Lennox, "Monte Carlo calculations to characterize the source for neutron therapy facilities," Med. Phys., vol. 26, pp. 783-792, 1999.

[15] GEANT4 Collaboration. [Online]. Available: http://wwwinfo.cern.ch/ asd/geant4/geant $4 . h$ html

[16] P. Bradley, A. Rosenfeld, K. Lee, D. Jamieson, and S. Satoh, "Charge collection and radiation hardness of a SOI microdosimeter for space and medical applications," IEEE Trans. Nucl. Sci., vol. 45, pp. 2700-2710, Dec. 1998.

[17] I. Cornelius, A. Rosenfeld, R. Siegele, and D. Cohen, "LET dependence of the charge collection efficiency of silicon microdosimeters," IEEE Trans. Nucl. Sci., vol. 50, pp. 2373-2379, Dec. 2003. 